\title{
Molluscum contagiosum: the rise and fall of imiquimod
}

\section{Editorial}

The trajectory of imiquimod began in 1997 when it first received approval from the US Food and Drug Administration (FDA) for the treatment of genital warts in adults. Soon, dermatologists and physicians adopted imiquimod as a viable option to treat molluscum contagiosum (MC) in children. Important to note that the off- label use of imiquimod was supported by small randomized controlled trials (RCT) and isolated case reports, besides the premise that the treatment would be successful because molluscum also represents a viral infection. However, such studies with small numbers of patients were not sufficient for the FDA. In 2006, the FDA requested 3 studies in order to evaluate the real effectiveness of imiquimod in the treatment of molluscum contagiosum in pediatric patients (2-12 years): 2 double-blind controlled and 1 pharmacokinetic. The results were not encouraging, since the efficacy of the topical application three times a week for up to 16 weeks has not been demonstrated. In one RCT, which enrolled 323 children, complete clearance at 18 weeks occurred in $24 \%$ of imiquimod-treated group and $26 \%$ of vehicle-treated group; another double-blind study, which enrolled 379 children, complete clearance occurred in $24 \%$ and $28 \%$, respectively. The author used exactly these words to describe the results: "The studies failed to demonstrate efficacy."

Furthermore, higher rates of lymphadenopathy, otitis media, conjunctivitis and local skin reactions (erythema was the most common) were observed in imiquimod-group. The study also highlights a search on AERS database that revealed nine cases of erythema multiform in adult patients and a case of Henoch-Scholein purpura in 3 years old boy treated for MC with imiquimod. Abnormal hematological findings were also more common in imiquimod group, leucopenia or neutropenia were associated to systemic effects of imiquimod. ${ }^{1}$ Curiously these two important RCTs have never been published. Only the pharmacokinetic study was published in $2008 .^{2}$ Therefore, these RCTs remained in a "literature limbus"; not even mentioned in the Cochrane systematic review of treatment for MC, until 2013. ${ }^{3}$ In 2015, this scenery changed when Kenneth A. Katz revealed these two unpublished $\mathrm{RCTs}^{4}$ and this year two reviews didn't recommended imiquimod as a routine treatment for MC. ${ }^{5,6} \mathrm{In}$ Brazil there's no RCTs data regarding imiquimod use, but according to my personal observation the use of imiquimod for MC increased over the past three years in my country and (unfortunately) imiquimod is often mentioned in continuing medical education courses as a viable alternative for the treatment of MC in children. This is the moment
Volume I Issue I - 2016

Felipe Ladeira de Oliveira

Department of Dermatology, Instituto Pos Graduacao Izamar Millidiu da Silva- IMS - Rio de Janeiro, Brazil

Correspondence: Felipe Ladeira de Oliveira, Dermatology, Instituto Pos Graduacao Izamar Millidiu da Silva- IMS - Rio de Janeiro, Brazil,Tel 21-993057370, Email oliveiraflmed@gmail.com

Received: September 14, 2016 | Published: October 05, 2016

for a change of attitude when prescribing, since I believe it is not valid to use a medicine that has no proven effectiveness. Purposely, this editorial has been written critically, aiming to guide the use of scientific knowledge in medical decisions.

\section{Acknowledgements}

None.

\section{Conflict of interest}

The author declares no conflict of interest.

\section{References}

1. Papadopoulos EJ. Clinical executive summary [Imiquimod]. 2016. p. 1-5.

2. Myhre PE, Levy ML, Eichenfield LF, et al. Pharmacokinetics and safety of imiquimod $5 \%$ cream in the treatment of molluscum contagiosum in children. Pediatr Dermatol. 2008;25(1):88-95.

3. Katz KA, Swetman GL. Imiquimod, molluscum, and the need for a better "best pharmaceuticals for children" act. Pediatrics. 2013;132(1):1-3.

4. Katz KA. Dermatologists, imiquimod and treatment of molluscum contagiosum in children: righting wrongs. JAMA Dermatol. 2015;151(2):125-126.

5. Rush J, Dinulos JG. Childhood skin and soft tissue infections: new discoveries and guidelines regarding the management of bacterial soft tissue infections, molluscum contagiosum, and warts. Curr Opin Pediatr. 2016;28(2):250-257.

6. Sterling J. Treatment of warts and molluscum: what does the evidence show? Curr Opin Pediatr. 2016;28(4):490-499. 